\section{Electromagnetic Effects Associated With Earthquakes and Volcanic Eruptions}

\section{PAGE 223}

Seismo-electromagnetic (SEM) effects are the electric and magnetic perturbations caused by the natural geophysical changes associated with earthquakes and volcanic eruptions. These changes appear to take place predominantly in the interval between a few hours and a few days before the originating shock, and they could therefore be used as short-term precursors when the phenomena involved are better understood. Traditionally, SEMs have stirred controversy among researchers. Understanding the generation mechanisms for these phenomena is difficult because a variety of very different physical processes are involved: plasma waves, wave propagation, rock physics, and seismology. Moreover, not all earthquakes and volcanic eruptions appear to produce such perturbations before their onset.

The two most popular hypotheses for the generation of electromagnetic emissions during earthquake processes involve either direct wave-production by compression of rocks near the focal point or electric discharges due to a redistribution of electric charges in the ground (piezoelectric or electrokinetic effects).

A joint symposium was held at the 1993 URSI General Assembly in Kyoto between the commissions on waves in plasmas, electromagnetic noise and interference, and ionospheric radio waves and propagation to review progress in the understanding of SEM effects. The symposium covered all aspects of the electromagnetic fields related to earthquakes and volcanic enuptions including measurements of electric and magnetic field changes; crust-generated electromagnetic emissions; anomalies in atmospheric electricity, ionospheric, and magnetospheric plasmas and in radio wave propagation; and laboratory experiments and theoretical models.
J. Y. Dea et al. presented results from a low frequency signatures monitoring network in Southern California. They determined the significance of anomalous events by comparing the background level in the 1 to $20 \mathrm{~Hz}$ region, and several examples of broadband precursor emissions were presented. A. C. Fraser-Smith discussed the search for ULF frequency magnetic precursor signals in California prior to moderately-large and large earthquakes $(M \geq 5)$. Following his latest observations, he suggested that there is a threshold of $M \sim 5$ for the magnitudes below which no magnetic field changes are observed, and that the changes can only be observed over distances of about 100-150 $\mathrm{km}$ from the epicenters.

SEM effects are also observed during and after earthquakes. T. Ondoh investigated observations of low frequency atmospherics associated with volcanic eruptions during nighttime. Statistical studies of ELF/VLF emissions recorded by low-altitude satellites during seismic events were independently analyzed by M. Parrot and O. A. Molchanov et al. The study by M. Parrot concerned data recorded by the AUREOL 3 satellite in intervals of \pm 24 hours around the time of 325 earthquakes of magnitude greater than 5 . It was shown that ELF waves related to seismic activity can be observed all along the magnetic meridian passing over the epicenter of an earthquake. The amplitude is a maximum when the difference between the longitude of the satellite and the longitude of the earthquake is less than $10^{\circ}$.

Slightly different results were obtained with the data of the INTERCOSMOS 24 satellite studied by Molchanov et al. Emissions were observed in two frequency bands: in the ELF range $(I<1000 \mathrm{~Hz})$, as in the previous presentation, but also in the VLF range, where the corresponding emissions could be observed far from the epicenter $\left(180^{\circ}\right)$ at the same $L$ value. Another statistical study with ground data was presented by T. Yoshino and I. Tomizawa. With a network recording emissions at $82 \mathrm{kHz}$, they reported the observation of 29 SEM events. They showed a relationship of the form $M=2.2+1.4 \log E$ between the earthquake magnitude $M$ and the distance $E$ (in $\mathrm{km}$ ) between epicenters and the receiver for SEM effects to be detected. But no relation was observed concerning the depth of the

et al. described a radio-tomographic method to investigate the ionospheric layers and therefore to detect anomalous changes prior to an earthquake. Using a satellite and several ground stations their method makes it possible to detect not only variations of the critical frequency but also variations of the ionospheric spatial structure. In the paper by A. M. Merzer, an explanation for the ULF magnetic field changes preceding the Loma Prieta earthquake was described. It was proposed that the anomalous changes were due to the formation of a long, thin, highly conductive region along the earthquake fault.

The two invited papers of the session were devoted to theoretical models that could explain the general SEM observations: $T$. Ishido reviewed electric and magnetic field generation in the Earth's crust by electrokinetic processes, and I. Yamada reported electromagnetic phenomena induced by rock fractures. In the poster session, $\mathrm{M}$. Hata and S. Yabashi showed observations of impulsive geomagnetic field variations before and after a series of earthquakes located at $10 \mathrm{~km}$ from their equipment. The emissions started 3 days before the first shock.

Following this URSI symposium, a workshop was held in Tokyo. A session covering SEM effects will take place during the next IAGA/IASPEI meeting in Boulder planned for July 3-14, 1995.-M. Parrot, LPCE/CNRS, France; $A$. C. Fraser-Smith, Stanford University, Calif.; O. A. Molchanov, Institute of Physics of the Earth, Moscow, and University of Electro-Communications, Japan; and T. Yoshino, University of Electro-Communications, Japan earthquakes. V. N. Oraevsky from the Desert Research Institute. Elachi has led the design and development of four major NASA systems carried aboard Seasat and three space shuttle missions in 1981, 1984, and 1994 The latest radar imaging system flown aboard the space shuttle has provided images of what is

\section{In Memoriam}

Robert S. Dietz died May 19, 1995, at age 80. He had been a member (Ocean Sciences) since 1947.

A. Wendell Engle died March 31, 1995, at age 78 . He was a retired life member (Seismology) who joined AGU in 1947.

Samuel Mandel died April 10, 1995, at age 77. He had been a member (Hydrology), since 1983.

Hiromi Niki died April 1, 1995, at age 58 since 1991 . He had been a member (Atmospheric Sciences)

\section{Honors}

Thomas J. Ahrens and Paul C. Jennings, both of the California Institute of Technology; Veerabhadran Ramanathan, of the University of California, San Diego; and Sean C. Solomon, of the Carnegie Institution of Washington have been elected as fellows to the American Academy of Arts and Sciences. Ahrens, Ramanathan, and Solomon were elected to the astronomy and Earth sciences section, while Jennings was elected to the educational and scientific administration section. believed to be the fabled lost city of Ubar under the sands of the Sahara Desert. Elachi is currently director for space and Earth science at the Jet Propulsion Laboratory.

\section{Recent Ph.D.s}

\section{Ocean Sciences}

Early sequence evolution during carbonate platform flooding: An actualistic model from 